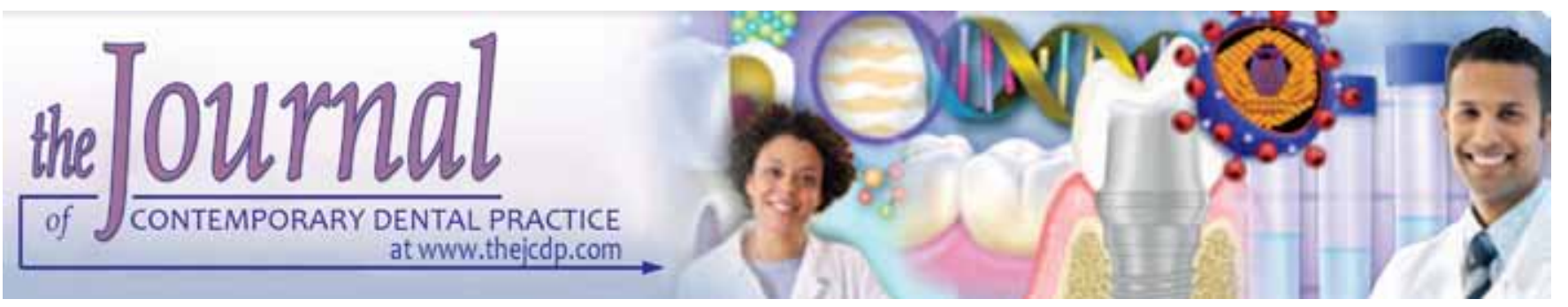

\title{
The Simultaneous Effect of Extended Etching Time and Casein Phosphopeptide-Amorphous Calcium Phosphate containing Paste Application on Shear Bond Strength of Etch-and-rinse Adhesive to Caries-affected Dentin
}

\footnotetext{
${ }^{1}$ Maryam Doozandeh, ${ }^{2}$ Maryam Firouzmandi, ${ }^{3}$ Milad Mirmohammadi
}

\begin{abstract}
Aim: This study evaluated the simultaneous effect of extended etching time and casein phosphopeptide-amorphous calcium phosphate (CPP-ACP) containing paste application on shear bond strength (SBS) of etch-and-rinse adhesive to cariesaffected-dentin (CAD).
\end{abstract}

Materials and methods: Fifty human third molars were used; 10 normal in group 1 and 40 caries-affected teeth in groups 2 to 5 . In the CAD groups, teeth were randomly assigned into four groups $(n=10)$. In groups 2 and 4, phosphoric acid etching for 15 and 45 seconds was used and in groups 3 and 5 , after 15 or 45 seconds etching respectively, CPP-ACP containing paste (MI paste) was applied for 3 minutes. After rinsing, single bond adhesive system and $Z_{250}$ composite were used for resin composite build-up in all groups. After storage, SBS test was measured at cross head speed of $0.5 \mathrm{~mm} / \mathrm{min}$. Data were analyzed using one/two-way ANOVA and Tukey HSD test $(\alpha=0.05)$.

Results: Normal dentin groups showed the highest SBS among different groups. There was a statistically significant difference between different etching times in CAD groups $(p<0.05)$, but there were no significant differences between SBS of SB adhesive to CAD with or without CPP-ACP pretreated in both etching times. Most of the failure modes were adhesive except in groups 1 and 5 which cohesive failure in composite was also observed.

${ }^{1,2}$ Department of Operative Dentistry, Shiraz University of Medical Sciences, Fars, Iran

${ }^{3}$ Department of Student Research Committee, Shiraz University of Medical Sciences, Fars, Iran

Corresponding Author: Maryam Firouzmandi, Assistant Professor, Department of Operative Dentistry, Shiraz University of Medical Sciences, Fars, Iran, Phone: 00987116263193 e-mail: firouzmandi@sums.ac.ir
Conclusion: The results of our study indicated that extended etching time up to 45 seconds could enhance the SBS of CAD and approach nearly to SBS of normal dentin. Moreover, MI paste pretreatment had not significant effect on SBS of etchand-rinse adhesive in CAD.

Clinical significance: Simultaneous application of extended etching time and CPP-ACP containing paste may be a suggested method in increasing the SBS of adhesive to CAD.

Keywords: Caries-affected-dentin, Casein-phosphopeptideamorphous calcium phosphate, Etching time, Shear bond strength.

How to cite this article: Doozandeh M, Firouzmandi M, Mirmohammadi M. The Simultaneous Effect of Extended Etching Time and Casein Phosphopeptide-Amorphous Calcium Phosphate containing Paste Application on Shear Bond Strength of Etch-and-rinse Adhesive to Caries-affected Dentin. J Contemp Dent Pract 2015;16(10):794-799.

Source of support: Nil

Conflict of interest: None

\section{INTRODUCTION}

Adhesive restorations are very popular by the clinicians in modern dentistry. ${ }^{1}$ Several studies analyzed the performance of adhesion mechanism in sound dentin. ${ }^{2,3}$ However, in conservative dentistry, dentists are usually involved with sclerotic dentin or caries-affected dentin (CAD), rather than normal dentin, in development of adhesive bond. ${ }^{4,5}$ Morphological and compositional changes in CAD influence its bond strength. In CAD, inter tubular dentin have lower mineral content and more porosity due to cyclic demineralization-remineralization caries process, so dentin is more permeable than normal dentin and results in easier and deeper diffusion of acidic conditioners and adhesive monomer. ${ }^{6,7}$ 
In addition, the dentinal tubules in CAD are occluded with acid resistance mineral crystals that limit the resin monomer infiltration and resin tag formation which decrease the adhesive resin retention. ${ }^{8}$

Several studies demonstrated that CAD has lower bond strength than normal dentin, either with totaletched or self-etched adhesives due to these biological conditions. ${ }^{6,9,10}$

Different methods had been suggested to increase the adhesive bond strength to CAD. Larissa Lenzi et al demonstrated that shortening of the etching time for etchand-rinse adhesives in primary teeth could improve the stability of resin-dentin bond in CAD. ${ }^{11}$ Another study showed that reducing the etching time (5 seconds) did not have any adverse effect on bond strength of Prime NT adhesive to CAD in primary teeth but acid etching for at least 10 seconds was more ideal for permanent teeth. ${ }^{12} \mathrm{On}$ the other hand, Arrais et al showed that additional and extended etching time could improve bonding to CAD with Clearfil SE bond and single bond by increasing the dissolution of mineral deposit in dentinal tubules and better infiltration of monomers into dentinal tubules and intertubular dentin. ${ }^{13}$

Recently, casein phosphopeptide-amorphous calcium phosphate (CPP-ACP) based compounds, such as MI paste (GC Corporation; Tokyo, Japan) have been introduced into the market. ${ }^{14}$ The presence of bioavailable calcium and phosphate in the CPP-ACP can maintain a supersaturated state in dental substrate ${ }^{15}$ Calcium and phosphate ions can easily diffuse into the porous lesion and deposite in the partially demineralized crystals and rebuild hydroxyl-apatite crystals. ${ }^{16}$ Several studies demonstrated that CPP-ACP could reduce demineralization and increase remineralization of dentin. ${ }^{17,18}$ Borges et al showed that active brushing of MI paste into normal dentin for 3 minutes before bonding procedure had no effect on bond strength of Clearfil SE Bond/Filtek $Z_{250}$ and Filtek P90 adhesive system/Filtek P90 composite, but improved the bond strength for Adper SE Plus/Filtek $\mathrm{Z}_{250}$ restorative system. ${ }^{19}$ Bahari et al revealed that CPP$\mathrm{ACP}$ paste pretreatment on CAD did not negatively affect on microtensile bond strength of single bond or clearfil SE bond, and single bond had higher bond strength to CAD than Clearfil SE Bond..$^{20}$ As mentioned previously, extended etching time could enhance the bond strength to CAD. ${ }^{13,21}$ However, because inter tubular dentin is previously hypomineralized in $\mathrm{CAD}$, extended etching could expose more collagen fibrils and make it more susceptible to hydrolytic degradation. ${ }^{22}$ The remineralizing ability of CPP-ACP can increase the mineral content of intertubular dentin and exposed collagen fibers; it is assumed that CPP-ACP paste pretreatment on CAD would enhance dentin bond strength and its durability. To the best of author's, knowledge, no study was found evaluated the combined effect of extended etching time and CPP-ACP paste pretreatment on CAD, Thus, the aim of this study was to evaluate the effect of this combination in the shear bond strength (SBS) of an etch-and-rinse adhesive system. The null hypothesis was that, extended etching and CPP-ACP application would have no effect on SBS of etch-and-rinse adhesive to CAD.

\section{MATERIALS AND METHODS}

Fifty human third molars (10 sound teeth and 40 teeth with occlusal caries)) were used in this study. The teeth were cleaned with a prophy-cup to remove gross debris then stored in a $1 \%$ chloramine $\mathrm{T}$ solution for 1 week, then placed in distilled water before the study. Before preparation, radiographic images of carious teeth were evaluated to confirm that the carious lesion extended about the half way to the pulp.

For normal dentin substrate, the enamel and dentin of occlusal surface were removed using a diamond bur (\#835/010; Teeskavan, Iran) in a low-speed handpiece under water coolant to achieve the mid-coronal sound dentin surfaces. For the teeth affected by caries, combination criteria of visual inspection, dental explorer and staining method was used to remove infected dentin. A caries detector solution (Snoop; pulpdent corporation, USA) was applied on the dentin surfaces for 10 seconds. After rinsing, the stained infected dentin was removed using a round carbide bur (\#C1SRA016; Jota, Swiss). This procedure was repeated until the dark stained cariesinfected dentin was removed and lightly stained cariesaffected dentin remained. The diameter of CAD area was measured by digital caliper (Mitutyo; Tokyo, Japan) to ensure that this area was not less than $3 \mathrm{~mm}$, essential for bonding procedure. A 600-grit silicon carbide paper under water was used to achieve a uniform dentin surfaces in all specimens.

The roots of all teeth were cut $3 \mathrm{~mm}$ below the CEJ and the teeth mounted in chemically-cured acrylic resin (Meliodent; Heraeus, Hanau, Germany).

Teeth with CAD substrate were then randomly assigned into four groups $(n=10)$ based on the etching time (15 vs 45 seconds), and surface treatment (with or without CPP-ACP). Flow Chart 1 demonstrates a diagrammatic representation of the experimental and control groups in this study.

In group 1 (ND, Control), the dentin surfaces were etched with $35 \%$ phosphoric acid (scotch bond universal Etchant, 3M ESPE; St Paul, USA) for 15 seconds, rinsed with water and blot dried with absorbent paper to keep 
Flow Chart 1: Diagrammatic representation of the experimental and control groups in this study

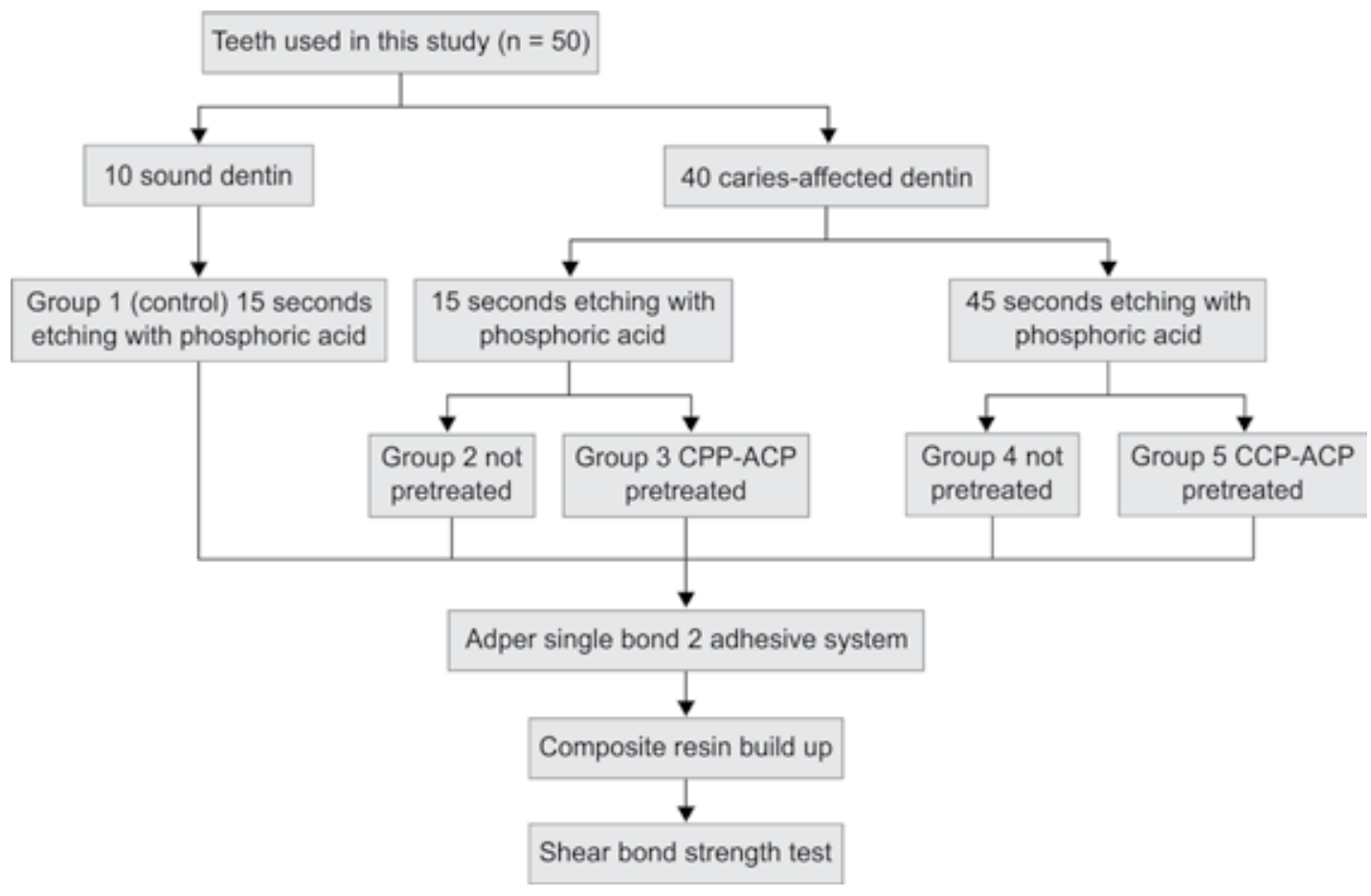

moist surfaces. Then two coats of an etch-and-rinsed adhesive, Adper single bond (SB), (3M ESPE; St Paul, USA) were applied on the surfaces, gently air dried, and light cured for 20 seconds (VIP Junior; Bisco, Schaumburg, USA) at $600 \mathrm{mw} / \mathrm{cm}^{2}$. Then, a plastic mold with $3 \mathrm{~mm}$ diameter and $2 \mathrm{~mm}$ height was placed on dentin surfaces and filled with $Z_{250}$ composite resin (3 m, ESPE; St Paul, USA), in $\mathrm{A}_{2}$ Shade and light cured with $600 \mathrm{mw} / \mathrm{cm}^{2}$ curing light.

In groups 2 and 4 , similar bonding procedures and composite buildups were done on CAD specimens, with the exception that the etching time in group 4 was extended to 45 seconds with $35 \%$ phosphoric acid instead of 15 seconds etching presented by the manufacturer in group 2.

In groups 3 and 5 , the specimens were etched for 15 or 45 seconds respectively, as described in previous groups, and then CPP-ACP containing paste (MI Paste; GC, Tokyo, Japan) was actively applied on the CAD specimens with a brush for 3 minutes. After rinsing the specimens with water, SB was applied and composite resin block were placed on the CAD surfaces according to the above mentioned protocol and polymerized.

After the bonding process, the specimens were stored in distilled water at $37^{\circ} \mathrm{C}$ for 24 hours. The specimens were mounted in a universal testing machine (Instron; Zwick, Germany) and shear forces were applied to each specimen at cross-head speed of $0.5 \mathrm{~mm} / \mathrm{min}$ at the interface between tooth and composite until failure occurred. The maximum load at failure was divided by the cross sectional area to determine SBS in MPa. The failure mode were observed under stereomicroscope at $40 \times$ magnification and categorized as:

Type A, cohesive failure in dentin, type $\mathrm{B}$, cohesive failure in composite, and type $C$, adhesive failure. Table 1 shows the composition of materials used in this study.

\section{STATISTICAL ANALYSIS}

The data of SBS in CAD groups were analyzed by twoway analysis of variance (ANOVA). One-way ANOVA also was used for analyzing the SBS differences between control (group 1) and CAD groups in different etching times (groups 2 and 4). Tukey HSD multiple comparison test was used to determine specific differences between that groups. Statistical significance was set at $p<0.05$. Statistical Package for Social Sciences Version 17 (Chicago, IL, USA) was used for statistical analysis.

\section{RESULTS}

The mean and standard deviation (SD) of SBS and failure modes in different groups are summarized in Table 2. The two-way ANOVA revealed that there was no significant interaction effect between application of CPP-ACP paste (use or nonuse) and the two time points $(p=0.439)$. So, the application of CPP-ACP on CAD did not have any significant effect on SBS of SB adhesive system. However, groups with 45 seconds etching time had significantly higher value of SBS $(27.25 \pm 1.68)$ compared with groups with 15 seconds etching time on CAD $(17.73 \pm 2.22)(p<0.001)$. The results of oneway ANOVA showed that increased etching time had 
Table 1: The composition of materials used in this study

\begin{tabular}{|c|c|c|c|}
\hline Materials & Composition & Manufacturer & Lot-number \\
\hline $\begin{array}{l}\text { Adper } \\
\text { single bond } \\
\text { adhesive }\end{array}$ & $\begin{array}{l}\text { 2-HEMA, Bis GMA, } \\
\text { Di-methacrylate, } \\
\text { amins, poly } \\
\text { alkenoic acid } \\
\text { copolymer, ethanol } \\
\text { water, camphor } \\
\text { quinone }\end{array}$ & $\begin{array}{l}\text { 3M, ESPE, } \\
\text { St Paul, MN, } \\
\text { USA }\end{array}$ & N 330309 \\
\hline $\begin{array}{l}\text { Filtek Z250 } \\
\text { composite }\end{array}$ & $\begin{array}{l}\text { Silane treated } \\
\text { silica, Bis-EMA, } \\
\text { UDMA, Bis-GMA, } \\
\text { TEGDMA }\end{array}$ & $\begin{array}{l}\text { 3M, ESPE, } \\
\text { St Paul, MN, } \\
\text { USA }\end{array}$ & N 144264 \\
\hline $\begin{array}{l}\text { Ml paste } \\
\text { (CPP-ACP } \\
\text { containing } \\
\text { paste) }\end{array}$ & $\begin{array}{l}\text { Glycerol, CPP- } \\
\text { ACP, D-sorbitol, } \\
\text { propylene glycol } \\
\text { silicon dioxide, } \\
\text { titanium dioxide }\end{array}$ & $\begin{array}{l}\text { GC } \\
\text { Corporation } \\
\text { Tokyo, Japan }\end{array}$ & $1306621 \mathrm{M}$ \\
\hline $\begin{array}{l}\text { Snoop caries } \\
\text { detector }\end{array}$ & $\begin{array}{l}\text { Dark blue dye in } \\
\text { propylene glycol } \\
\text { liquid formulation }\end{array}$ & $\begin{array}{l}\text { Pulpdent } \\
\text { Corporation } \\
\text { USA }\end{array}$ & 140218 \\
\hline
\end{tabular}

Table 2: Mean and standard deviation (SD) of shear bond strength values (MPa) and types of failure modes in different groups

\begin{tabular}{|c|c|c|c|c|c|}
\hline \multirow[b]{2}{*}{ Groups } & \multicolumn{2}{|c|}{$\begin{array}{l}\text { Shear bond strength } \\
(\mathrm{MPa})\end{array}$} & \multicolumn{3}{|c|}{ Type of failure modes } \\
\hline & Mean & $S D$ & $A$ & $B$ & $C$ \\
\hline 1 & 28.44 & 3.63 & 2 & 4 & 4 \\
\hline 2 & 16.98 & 1.83 & 0 & 0 & 10 \\
\hline 3 & 18.48 & 2.40 & 0 & 0 & 10 \\
\hline 4 & 26.98 & 1.90 & 0 & 1 & 9 \\
\hline 5 & 27.52 & 1.49 & 1 & 5 & 4 \\
\hline
\end{tabular}

significant effect on SBS of CAD (group 2 vs group 4) $(\mathrm{p}<0.05)$. TukeyHSD multiplecomparison testalso demonstrated that the SBS of group 1 (ND) was significantly higher than group 2, but there was not any significant differences between groups 1 and 4 .

As presented in Table 2, the predominant failure modes in groups 2, 3 and 4 were adhesive, but in groups 1 and 5 cohesive failures in dentin and composite were also observed.

\section{DISCUSSION}

In order to ensure the initial and long-term success of adhesive restorations, the adhesive systems must have adequate bonding performance on all types of dentin substrates. By development of conservative dentistry, CAD may be used as a substrate for bonding in addition to ND. ${ }^{13}$ The results of our study showed that the CAD had lower SBS compared to ND. This result was inconsistent with other studies. ${ }^{19,23,24,25}$ As mentioned previously, the structure of CAD is altered which cause poor hybridization and can be responsible for reduced bond strength. Xuan et al showed that there was no significant difference in bond strength of $\mathrm{SB}$ adhesive to $\mathrm{ND}$ and $\mathrm{CAD}{ }^{26}$
The intermittent cycles of demineralization and remineralization during carious process cause the orifices of dentinal tubules to be closed by larger and more acidresistant calcium-phosphate crystals. ${ }^{23}$ So, in the present study, we increased the etching time of CAD to 45 seconds and significant increase in SBS was observed which was nearly close to SBS of ND. Arrais et al also demonstrated that additional and extended etching time could improve bonding of Clearfil SE Bond and SB adhesive to CAD.

In that study, SEM analysis had revealed that although over-etching could not completely remove the mineral crystals in dentinal tubules, the partial removal of acidresistant deposits might increase the permeability of dentinal tubules. Therefore, the funnel shaped resin tags were formed, together with the infiltration of resin into lateral branches (resin micro-tag), contributed to enhance the bond strength in CAD. ${ }^{13,27}$

Although the extended etching time could enhance the SBS in CAD, this was also lead to deeper dentin demineralization in sound dentin that surrounds the $C A D$ and especially the inter tubular dentin which is already hypomineralized. The adhesive monomer may not be able to totally penetrate into this deep demineralized layer. Several studies revealed that the presence of monomerfree collagen fibril at the base of hybrid layer may be prone to hydrolytic degradation and would compromise the long-term durability of adhesive procedure. ${ }^{22,28}$

The novel recaldent technology, has the capacity to remineralize CAD. ${ }^{16,29}$ Therefore, in our study, we pretreated the CAD surfaces with CPP-ACP paste after acid-etching. We assumed that the increased mineral content of inter and peritubular dentin might compensate for the side effects of over-etching on resin-dentin bond strength. The results showed that 3 minutes CPP-ACP pretreatment of $C A D$ after etching did not have any significant effect on SBS of SB adhesive either with 15 or 45 seconds etching time. Borges et al found that this pretreatment did not have any negative effect on push out bond strength of two self-etch adhesives and also improve the bond strength of Adper Self Etch plus adhesive because of the increased mineral content. ${ }^{19}$ Adebayo et al found that dentin preconditioning with CPP-ACP paste (60 minutes per day for 7 days) did not show any adverse effect on the resin-dentin morphology and $\mu$ SBS of Clearfil SE Bond or G bond in the presence of smear layer. ${ }^{30,31}$ Both previous studies evaluated the effect of CPP-ACP paste on bonding to ND. Bahari et al showed that 5 consecutive days of $\mathrm{CPP}-\mathrm{ACP}$ application for 15 minutes did not have any significant effect on bond strength of SB or Clearfil SE bond to CAD. ${ }^{20}$

Failure mode analysis in our study showed that although MI paste treatment had no effect on SBS of SB 
after 45 seconds etching compared to no treatment group, most of the failures were cohesive in composite resin. Therefore, it might be concluded that this pretreatment have beneficial effect on adhesive interface of CAD.

In this study, we actively treated CAD surfaces for 3 minutes immediately after etching that can be more feasible for dentists. As described before, it had no negative effect on SBS of SB. Yang et al also demonstrated that calcium-containing desensitizers like CPP-ACP could create effective tubular occlusion without affecting the microtensile bond strength after etching in etch and rinse adhesives. $^{32}$

Further long-term studies plus SEM analysis should be performed to assess the effect of extended or additional etching and the usage of CPP-ACP paste on bond strength of both etch- and -rinse and self-etch adhesives to CAD.

\section{CONCLUSION}

Within the limitations of this study, we could conclude that:

- Shear bond strength of single bond adhesive to ND was higher than CAD

- Extended etching time could enhance the SBS of single bond adhesive to CAD and nearly close to SBS of ND

- Application of CPP-ACP paste prior to bonding procedure did not have any significant effect on SBS of etch-and-rinse adhesive to CAD neither with 15 seconds nor with 45 seconds etching time.

\section{ACKNOWLEDGMENTS}

The authors thank the Vice-Chancellory of Shiraz University of Medical Science for supporting this research (Grant\# 8793101). This article is based on the thesis by Dr M Mirmohammadi. The authors also thank Dr Vosoug of the dental research development center, of the school of Dentistry for the statistical analysis and Dr Sh Hamedani (DDs, MSc) for his editorial assistance.

\section{REFERENCES}

1. Estafan D, Klodnitskaya L, Wolff M. Treatment planning in esthetic dentistry requires careful listening to the patient. Gen Dent 2008;56(3):290-292.

2. Giannini M, Carvalho RM, Martins LR, Dias CT, Pashley DH. The influence of tubule density and area of solid dentin on bond strength of two adhesive systems to dentin. Adhes Dent 2001;3(4):315-324.

3. Pereira PN, Okuda M, Sano H, Yoshikawa T, Burrow MF, Tagami J. Effect of intrinsic wetness and regional difference on dentin bond strength. Dent Mater 1999;15(1):46-53.

4. Eick JD, Gwinnett AJ, Pashley DH, Robinson SJ. Current concepts on adhesion to dentin. Crit Rev Oral Biol Med 1997; 8(3):306-335.
5. Marshall GW Jr, Marshall SJ, Kinney JH, Balooch M. The dentin substrate: structure and properties related to bonding. J Dent 1997;25(6):441-458.

6. Yoshiyama M, Tay FR, Doi J, Nishitani Y, Yamada T, Itou K, et al. Bonding of self-etch and total-etch adhesives to carious dentin. J Dent Res 2002;81(8):556-560.

7. Haj-Ali R, Walker M, Williams K, Wang Y, Spencer P. Histomorphologic characterization of noncarious and caries-affected dentin/adhesive interfaces. J Prosthodont 2006;15(2):82-88.

8. Nakajima M, Sano H, Burrow MF, Tagami J, Yoshiyama M, Ebisu S, et al. Tensile bond strength and SEM evaluation of caries-affected dentin using dentin adhesives. J Dent Res 1995;74(10):1679-1688.

9. Koyuturk AE, Sengun A, Ozer F, Sener Y, Gokalp A. Shear bond strengths of self-etching adhesives to caries-affected dentin on the gingival wall. Dent Materials J 2006;25(1):59-65.

10. Wei S, Sadr A, Shimada Y, Tagami J. Effect of caries-affected dentin hardness on the shear bond strength of current adhesives. Adhesive Denti 2008;10(6):431-440.

11. Lenzi TL, Braga MM, Raggio DP. Shortening the etching time for etch-and-rinse adhesives increases the bond stability to simulated caries-affected primary dentin. J Adhes Dent 2014;16(3):235-241.

12. Scheffel DL, Ricci HA, de Souza Costa CA, Pashley DH, Hebling J. Effect of reducing acid etching time on bond strength to noncarious and caries-affected primary and permanent dentin. Pediatr Dent 2013;35(7):199-204.

13. Arrais CA, Giannini M, Nakajima M, Tagami J. Effects of additional and extended acid etching on bonding to cariesaffected dentine. Eur J Oral Sci 2004;112(5):458-464.

14. Cross KJ, Huq NL, Reynolds EC. Casein phosphopeptides in oral health-chemistry and clinical applications. Current Pharmaceutical Design 2007;13(8):793-800.

15. Reynolds EC. Anticariogenic complexes of amorphous calcium phosphate stabilized by casein phosphopeptides: a review. Special care in dentistry: official publication of the Amer Assoc Hospital Dent, Acad Dent for the Handicapped, and the Am Soc Geriatric Dent 1998;18(1):8-16.

16. Kumar VL, Itthagarun A, King NM. The effect of casein phosphopeptide-amorphous calcium phosphate on remineralization of artificial caries-like lesions: an in vitro study. Aust Dent J 2008;53(1):34-40.

17. Yamaguchi $\mathrm{K}$, Miyazaki M, Takamizawa $\mathrm{T}$, Inage $\mathrm{H}$, Kurokawa H. Ultrasonic determination of the effect of casein phosphopeptide-amorphous calcium phosphate paste on the demineralization of bovine dentin. Caries Res 2007;41(3): 204-207.

18. Rahiotis C, Vougiouklakis G. Effect of a CPP-ACP agent on the demineralization and remineralization of dentine in vitro. J Dentist 2007;35(8):695-698.

19. Borges BC, Souza-Junior EJ, da Costa Gde F, Pinheiro IV, Sinhoreti MA, Braz R, et al. Effect of dentin pretreatment with a casein phosphopeptide-amorphous calcium phosphate paste on dentin bond strength in tridimensional cavities. Acta Odontologica Scandinavica 2013;71(1):271-277.

20. Bahari M, Oskoee SS, Kimyai S, Pouralibaba F, Farhadi F, Norouzi M. Effect of casein phosphopeptide-amorphous calcium phosphate treatment on microtensile bond strength to carious affected dentin using two adhesive strategies. J Dent Res Dent Clin Dent Prospects 2014;8(3):141. 
21. Gwinnett AJ. Quantitative contribution of resin infiltration/ hybridization to dentin bonding. Am J Dent 1993;6(1):7-9.

22. Burrow MF, Tagami J, Hosoda H. The long-term durability of bond strengths to dentin. The Bulletin of Tokyo Medical and Dental University 1993;40(4):173-191.

23. Nakajima N, Sano H, Urabe I, Tagami J, Pashley D. Bond strengths of single-bottle dentin adhesives to caries-affected dentin. Oper Dent 2000;25:2-10.

24. Yoshiyama M, Urayama A, Kimochi T, Matsuo T, Pashley DH. Comparison of conventional vs self-etching adhesive bonds to caries-affected dentin. Oper Dent 2000;25(3):163-169.

25. Nakajima M, Kunawarote S, Prasansuttiporn T, Tagami J. Bonding to caries-affected dentin. Japanese Dent Sci Rev 2011;47(2):102-114.

26. Xuan W, Hou BX, Lu YL. Bond strength of different adhesives to normal and caries-affected dentins. Chinese Med J 2010;123(3):332-336.

27. Prati C, Chersoni S, Mongiorgi R, Pashley DH. Resininfiltrated dentin layer formation of new bonding systems. Oper Dent 1998;23(4):185-194.
28. Hashimoto M, Ohno H, Kaga M, Endo K, Sano H, Oguchi H. In vivo degradation of resin-dentin bonds in humans over 1 to 3 years. J Dent Res 2000;79(6):1385-1391.

29. Andersson A, Skold-Larsson K, Hallgren A, Petersson LG, Twetman S. Effect of a dental cream containing amorphous cream phosphate complexes on white spot lesion regression assessed by laser fluorescence. Oral Health and Prevent Dentist 2007;5(3):229-233.

30. Adebayo OA, Burrow MF, Tyas MJ. Resin-dentine interfacial morphology following CPP-ACP treatment. J Dent 2010;38(2):96-105.

31. Adebayo OA, Burrow MF, Tyas MJ. Dentine bonding after CPP-ACP paste treatment with and without conditioning. J Dent 2008;36(12):1013-1024.

32. Yang H, Pei D, Chen Z, Lei J, Zhou L, Huang C. Effects of the application sequence of calcium-containing desensitising pastes during etch-and-rinse adhesive restoration. J Dent 2014; 42(9):1115-1123. 